\title{
Rare-Gas Clusters in Intense Extreme-Ultraviolet Pulses from a High-Order Harmonic Source
}

\author{
B. Schütte, ${ }^{1, *}$ M. Arbeiter, ${ }^{2}$ Th. Fennel, ${ }^{2}$ M. J. J. Vrakking, ${ }^{1}$ and A. Rouzée ${ }^{1}$ \\ ${ }^{1}$ Max-Born-Institut, Max-Born-Strasse 2A, 12489 Berlin, Germany \\ ${ }^{2}$ Institute of Physics, University of Rostock, Universitäsplatz 3, 18051 Rostock, Germany
}

(Received 12 November 2013; published 20 February 2014)

\begin{abstract}
We report evidence for two previously unidentified effects in the ionization of rare-gas clusters by intense extreme-ultraviolet pulses. First, electron spectra indicate multistep photoemission with increasing isotropy for larger clusters due to electron-atom collisions. Second, very slow (meV) electrons are interpreted as the first experimental evidence for Rydberg-like atomic state formation in the nanoplasma expansion. Only small fractions of $\mathrm{Xe}^{2+}$ ions were found, in sharp contrast to previous results recorded under comparable conditions [Murphy et al., Phys. Rev. Lett. 101, 203401 (2008)].
\end{abstract}

In the last decade, tremendous development of novel ultrafast light sources in the short-wavelength range has significantly deepened our understanding of laser-matter interactions on ultrashort time and length scales. Freeelectron lasers (FELs) such as FLASH [1] and LCLS [2] have opened up a new regime of high-brilliance experiments in the vacuum-ultraviolet (VUV), extremeultraviolet (XUV), and x-ray domains, with the promise of significant applications in biology and chemistry. Being a prerequisite for these developments, the physics of high intensity laser-matter interactions at short wavelength is a current topical issue [3,4].

High-order harmonic generation (HHG) sources provide VUV, XUV, and x-ray pulses with lower fluence than FELs but offer very short pulses down to the attosecond regime [5], with large-wavelength tunability [6], and the straightforward setup of two-color pump-probe experiments with high time resolution. Moreover, HHG-based pulses are fully coherent, avoiding statistical pulse structure effects stemming from the self-amplified spontaneous emission process at FELs [7]. Because of recent increases in the flux of HHG sources up to the $\mu \mathrm{J}$ level [8], it has become possible to study VUV- or XUV-induced multiphoton ionization processes in a laboratory-scale environment [9]. At wavelengths $\lambda \geq 30 \mathrm{~nm}$, the experimentally achieved peak intensities [10,11] are competitive with FELs [12].

The interest in XUV-induced dynamics of rare-gas clusters results from the radically different response when compared to intense near-infrared (NIR) fields, where field-driven avalanching, nonlinear nanoplasma heating, and collective resonance effects play a crucial role [13,14]. In the XUV (and x-ray) range, laser absorption proceeds dominantly via atomic single-photon ionization while collisional laser heating of the nanoplasma remains negligible up to high intensities [15]. Sequential direct photoemission (multistep ionization) leads to characteristic plateau-shaped electron energy spectra due to energy shifts induced by the transient cluster Coulomb potential buildup [16]. After sufficient outer ionization, electron trapping frustrates direct photoemission and a nanoplasma is formed, and only evaporative emission of quasifree electrons from the expanding cluster remains operational for sufficiently intense laser fields [15,17]. The wellcontrolled and tunable nanoplasma generation makes clusters under intense short-wavelength fields ideal model systems for studying ultrafast relaxation processes in highly excited many-particle systems [18-21].

In this Letter, we employ velocity map imaging (VMI) [22] to record angular-resolved ion and electron spectra for Xe and Ar clusters exposed to intense XUV pulses generated in a laser-based high-harmonic source. The observed size dependence of the electron energy spectra can be well explained by sequential multicolor multistep ionization induced by the dominant harmonic orders of the pulses. We find that the angular distribution of electrons changes from strongly anisotropic for atoms and small clusters to almost isotropic for larger clusters, revealing the increasing significance of elastic electron-atom collisions. Moreover, we find a strong emission of very slow electrons in the meV range that supports the idea of frustrated recombination [23], where the detector electric field (in this case, the extraction field of the VMI spectrometer) reionizes high-lying Rydberg states generated during the nanoplasma expansion, thereby increasing the ion and electron signals. Finally, we address the existing discrepancy between results from rare-gas cluster experiments at FEL sources $[12,16]$ and HHG sources $[24,25]$. In the former case, atomic ions in moderate ionization stages were observed for irradiation intensities around $10^{13} \mathrm{~W} / \mathrm{cm}^{2}$. For example, in studies at the SPring-8 Compact SASE Source, $\mathrm{Xe}^{n+}$ ions up to $n=4$ were found after exposing $\mathrm{Xe}_{N}$ clusters $(\langle N\rangle=2000-50000)$ to 100 fs FEL pulses with $\lambda=52 \mathrm{~nm}$ pulses at an intensity of 
$2 \times 10^{13} \mathrm{~W} / \mathrm{cm}^{2}$ [12]. In contrast, HHG experiments [24] at intensities 2-3 orders of magnitudes lower indicated charge states up to $\mathrm{Xe}^{8+}$. In the present study, we obtain results that are very different from Ref. [24] and fully consistent with the FEL experiments [12] and corresponding numerical models [20].

For the experiments, we employ 32 fs NIR pulses from a Ti:sapphire amplifier with $790 \mathrm{~nm}$ central wavelength, $50 \mathrm{~Hz}$ repetition rate, and $35 \mathrm{~mJ}$ maximum pulse energy [26]. The NIR beam is focused into the HHG chamber by a spherical mirror with $5 \mathrm{~m}$ focal length, and the HHG conversion process takes place in a $15 \mathrm{~cm}$ long gas cell that is statically filled with $\mathrm{Xe}$ or $\mathrm{Ar}$ at a pressure of 1.2 or 7 mbar, respectively. The collinear NIR and XUV beams propagate for another $5 \mathrm{~m}$ towards a spectrometer chamber where an iris blocks the outer part of the NIR beam, while the XUV beam is transmitted. A $200 \mathrm{~nm}$ thick aluminum filter attenuates the remaining NIR light overlapping the XUV by several orders of magnitude. To confirm that the residual NIR light does not influence the experimental results, two silicon mirrors can be added under Brewster's angle for further NIR attenuation by 3 orders of magnitude. We checked that when adding the silicon mirrors, the spectra did not change substantially, except for a global signal reduction due to the reduced XUV transmission. The measured HHG pulse energy of $200 \mathrm{~nJ}$ (obtained from a calibrated XUV photodiode AXUV100) after the filter supports pulse energies at the source at the microjoule level. For spectral characterization, a grating is moved into the beam path to disperse the XUV pulses onto a multichannelplate and phosphor screen assembly.

Upon removal of the grating, the beam propagates towards the experimental chamber, which includes a VMI spectrometer and a cluster source. The HHG beam is focused into the interaction zone and spectrally selected by a spherical AlMg-SiC multilayer mirror with a focal length of $75 \mathrm{~mm}$. The energy in the interaction zone is up to $10 \mathrm{~nJ}$, and the focal radius is estimated to be $3 \mu \mathrm{m}$, leading to an intensity at the focus of $2 \times 10^{12} \mathrm{~W} / \mathrm{cm}^{2}$, assuming a $15 \mathrm{fs}$ pulse duration. The XUV beam is intersected at right angles by a cluster beam produced by a piezoelectric valve with a $0.5 \mathrm{~mm}$ diameter cylindrical nozzle. The cluster size is controlled by changing the backing pressure between 2 and 20 bar. We used the Hagena scaling law [27] to calculate the average number of atoms per cluster. A molecular beam skimmer with $0.5 \mathrm{~mm}$ diameter selects the central part of the cluster beam for use in the VMI detector. Since the interaction zone has a length of $0.7 \mathrm{~mm}$ and the Rayleigh length of the XUV beam is estimated as $0.1 \mathrm{~mm}$, this leads to an averaging over different intensities in the experiment. An electric field of up to $2 \mathrm{kV} / \mathrm{cm}$ accelerates the electrons and ions generated by cluster ionization of the XUV pulse towards a multichannel-platephosphor screen assembly, where it is possible to detect the flight times of the ions. Alternatively, two-dimensional projections of the ion or electron momentum distributions are recorded using a charge-coupled device camera. Using an Abel inversion, the 3D momentum distribution is retrieved and enables us to extract the angular-resolved kinetic energy spectra [28].

As a starting point, we compare time-of-flight ion spectra from Xe atoms and Xe clusters $(\langle N\rangle=36000)$ in Fig. 1(a), thus allowing direct comparison with time-of-flight spectrometry results from previous VUV and XUV cluster experiments $[12,16,24,29,30]$. Here, the HHG medium was Xe with a cutoff energy in the HHG spectrum below the reflectivity maximum of the multilayer mirror at $32 \mathrm{eV}$. As a result, clusters were mainly excited by the 13 th and 15 th harmonics, and the intensity of the XUV pulse is estimated as $I=5 \times 10^{11} \mathrm{~W} / \mathrm{cm}^{2}$. While only peaks corresponding to $\mathrm{Xe}^{+}$and $\mathrm{Xe}^{2+}$ ions are observed for atoms, two new contributions from $\mathrm{Xe}_{2}^{+}$and $\mathrm{Xe}_{3}^{+}$appear in the cluster results. The clearly observable reduced fraction of doubly charged ions in the cluster results supports charge-state lowering via recombination processes, as predicted previously by simulations $[18,20]$.

Our measurements are in sharp contrast to results obtained in the previous HHG experiment [24], where charge states up to $\mathrm{Xe}^{8+}$ were reported for intensities of only $5 \times 10^{10} \mathrm{~W} / \mathrm{cm}^{2}$. We estimate that under these conditions, only about 1 in 1000 atoms absorbs a photon, in agreement with Ref. [31], making the observation of $\mathrm{Xe}^{8+}$ difficult to rationalize. By contrast, calculations for $\mathrm{Ar}_{923}$ clusters at $38 \mathrm{eV}$ photon energy only predict charge states up to $4+$ for similar pulse durations but at much higher


FIG. 1 (color online). (a) Ion time-of-flight spectra from Xe atoms and $\mathrm{Xe}_{N}$ clusters with $\langle N\rangle=36000$ after irradiation by an intense XUV pulse created by $\mathrm{HHG}$ in $\mathrm{Xe}$ and containing predominantly the harmonics 13 and 15 (with photon energies of 20.5 and $23.6 \mathrm{eV}$ ). (b) Kinetic energy spectra and (c)-(e) charge-state-resolved velocity map images of ions from Xe clusters. Ring structures are observed with a decreasing radius for larger $m / q$ ratios. The black curve in (b) shows the $\mathrm{Xe}^{2+}$ signal smoothed over a $5 \mathrm{eV}$ range. 
intensities $\left(10^{13} \mathrm{~W} / \mathrm{cm}^{2}\right)$ [20]. These experimental and numerical results cannot be reconciled.

In Figs. 1(c)-1(e), we present charge-state-resolved photoion momentum spectra recorded with the VMI. The doubly charged ions show a ring structure, similar to previous FEL results reported by Iwayama and coworkers [12], who attributed this feature to surface ion emission from a nanoplasma generated via frustrated ionization. A comparison of the kinetic energy distributions shown in Figs. 1(c)-1(e) and summarized in Fig. 1(b) shows qualitative differences between $\mathrm{Xe}^{+}$and $\mathrm{Xe}^{2+}$. While the $\mathrm{Xe}^{2+}$ distribution exhibits a broad peak with a maximum around $50 \mathrm{eV}$ (N.B., the low energy feature is ascribed to uncondensed atoms), $\mathrm{Xe}^{+}$shows a smooth, exponential-shaped distribution. These two features agree well with ion energy spectra from molecular dynamics simulations of highly frustrated XUV cluster ionization [20]. Partial ionization of the cluster atoms is signified by the presence of considerable signal from $\mathrm{Xe}_{2}^{+}$. Our ion spectra are therefore fully compatible with theory and previous FEL measurements.

While the ion energy spectra reflect the long-term evolution of the cluster expansion, photoelectron spectra contain information on the microscopic ionization dynamics during the laser excitation. For measuring photoelectron spectra, we used Ar as the HHG medium (allowing for the generation of higher photon energies) and as the target material as well. In Fig. 2, we depict photoelectron spectra from $\mathrm{Ar}$ atoms and $\mathrm{Ar}_{N}$ clusters. Compared to $\mathrm{Xe}$, the assignment of spectral features to the relevant electronic states is simpler in Ar because of the significantly smaller spin-orbit splitting. We note, however, that the results obtained in Xe show the same qualitative behavior compared to the results presented here. As is evident from Figs. 2(a) and 2(e), atoms are predominantly ionized by the 21 st harmonic with additional contributions mainly from lower harmonics. The projected momentum distribution in Fig. 2(a) shows the pronounced anisotropy of the atomic photolines for each contributing harmonic.

The evolution of the 2D photoelectron momentum distributions in Fig. 2(a)-2(d) shows two pronounced effects when going from atoms to clusters. First, the anisotropy of the photolines is reduced for larger clusters. Second, an additional continuous contribution appears between the photolines, increasing with cluster size and developing to the dominant feature for the largest cluster size. Analyzed in terms of angle-integrated kinetic energy distributions, cf. Figs. 2(e) and 2(f), the sequence of wellisolated photolines observed with atoms evolves to a plateaulike spectrum with maximum yields at low energy for the largest clusters.

To model the photoemission, we generalized our previously developed Monte Carlo simulation model $[15,16]$ to HHG pulses. Therefore, we consider sequential multicolor multistep photoemission according to instantaneous


FIG. 2 (color online). (a)-(d) Velocity map images of electron distributions from Ar atoms and clusters with different sizes after dominant irradiation with the 21st harmonic generated in Ar. (e)-(h) The corresponding angular-integrated kinetic energy spectra show a good agreement with Monte Carlo calculations that were carried out at intensities of $5 \times 10^{11}, 1 \times 10^{12}$, and $2 \times 10^{12} \mathrm{~W} / \mathrm{cm}^{2}$. (i)-(l) The experimental spectra for parallel and perpendicular emission smoothed over $0.5 \mathrm{eV}$ reveal a transition from a strongly anisotropic to an isotropic distribution for larger clusters. The arrows in (j) indicate bulklike peaks that become visible in the perpendicular emission spectra.

atomic ionization probabilities calculated from the intensity profiles of a set of Gaussian pulses, each corresponding to one of the harmonics in the experimental XUV field. The single-particle energy $E_{\mathrm{SP}}$ of the photoemitted electrons consists of the excess energy from photoabsorption and the attractive cluster Coulomb potential resulting from previous ionization events. In the simulation, only ionization events with $E_{\mathrm{SP}}>0$ are considered and contribute to the electron emission spectra. The cluster geometries are described by icosahedral structures scaled to the density of solid Ar. Since ionic motion is negligible for the considered pulse durations, the cluster atoms are fixed during the XUV excitation. The spatial intensity profile of the experiment is accounted for via three-dimensional focal volume averaging using the experimental focus parameters. The cluster size distribution is included by a weighted averaging with a log-normal size distribution. Calculated electron kinetic energy spectra for three different laser intensities are compared to the experimental angleintegrated data in Figs. 2(e)-2(h). The simulated spectra are normalized using the peak of the experimental photoline near $17 \mathrm{eV}$ (21st harmonic) and broadened by 
convolution with a Gaussian function $(\sigma=0.29 \mathrm{eV})$ to match the width of the experimental atomic photolines in Fig. 2(e).

With increasing cluster size, the simulated spectra show (i) increasing electron signal between the photolines and (ii) a global increase of the spectra towards lower energy, similar to the experiment. Note that the signal increase in the low-order photolines with cluster size is also well reproduced by the calculations. Best agreement with the experimental spectra is found in the intensity range $(1-2) \times 10^{12} \mathrm{~W} / \mathrm{cm}^{2}$, compatible with the estimated intensity of the experiment. Remaining deviations are attributed to contributions from uncondensed atoms in the beam and the neglect of inelastic collisions in the modeling. However, all qualitative features of the spectra can be well explained by the multicolor multistep ionization model. In particular, we note that the increase of the electron signal towards low energy for the large clusters can only be explained by considering the instantaneous presence of multiple harmonic orders. In a simplified picture, each harmonic order generates a multistep plateau with the corresponding upper cutoff energy, leading to signal accumulation at low energy.

Experimental electron spectra parallel and perpendicular to the XUV polarization are presented in Figs. 2(i)-2(l), where a strong anisotropy is found in the atomic case [Fig. 2(i)]. The perpendicular emission spectrum for small clusters with $\langle N\rangle=1000$ shows new peaks at about $1 \mathrm{eV}$ higher kinetic energies compared to the atomic spectra [see arrows in Fig. 2(j)]. These peaks are identified as bulk electrons from the inner part of the cluster [32]. While the bulk contributions are more easily identified in the perpendicular emission spectrum, they are also visible in the parallel spectra as a shoulder on the atomic lines which increases for larger clusters. Importantly, the bulk peaks have an isotropic distribution emphasizing their origin from the cluster core. These additional contributions were not previously observed in FEL experiments (see, e.g., Ref. [16]) and demonstrate the increased resolution of the HHG source. Note that a weak contribution from condensation is also seen in the atomic spectrum.

When comparing the parallel and perpendicular emission spectra of clusters [Figs. 2(j)-2(1)], an increasingly isotropic emission is observed for increasing cluster sizes, in agreement with previous theory results [33]. We attribute this to the longer average escape path of direct photoelectrons in larger clusters that increases the probability for electron-atom collisions. Note that evaporative electron emission from a nanoplasma would have an isotropic distribution located at low kinetic energies. However, it is not easily possible to distinguish between electron contributions from multistep and evaporative emission.

A striking feature of the VMI spectra in Figs. 2(b)-2(d) is the strong central contribution corresponding to extremely low kinetic energies which is absent for atoms. We


FIG. 3 (color online). (a) Electron kinetic energy spectrum for Ar clusters with $\langle N\rangle=3500$ highlighting the low energy electrons attributed to the ionization of Rydberg atoms by the detector electric field. The data are normalized and show that the average energy increases for larger field strengths. (b) The complete electron kinetic energy distribution spectrum for different field strengths (note the difference in the vertical scale). The inset shows a kinetic energy spectrum obtained after cluster ionization with $790 \mathrm{~nm}$ pulses at $5 \times 10^{13} \mathrm{~W} / \mathrm{cm}^{2}$.

interpret this signal as arising from quasifree electrons that occupy high-lying Rydberg states during the nanoplasma expansion, as investigated theoretically for the case of cluster ionization with NIR pulses [23]. In Fig. 3(a), a zoom at low kinetic energies shows that the electrons have energies in the range of a few $\mathrm{meV}$ to a few tens of $\mathrm{meV}$. By increasing the detector electric field strength, the average kinetic energy of the electrons increases, underscoring the role of the dc electric field in the ionization process of Rydberg atoms. For comparison, the spectra at larger energies in Fig. 3(b) do not depend on the applied field strength. Hence, these results are a strong indication for the generation of Rydberg atoms in the nanoplasma due to collisional recombination and their subsequent reionization by the detector electric field. We would not expect such a characteristic emission of very slow electrons from other mechanisms such as evaporative emission from the nanoplasma or Rydberg-Rydberg interactions. In order to demonstrate that the observed process is not limited to XUV excitation wavelengths, an electron kinetic energy spectrum for the case of cluster ionization with a $790 \mathrm{~nm}$ NIR pulse is shown in the inset of Fig. 3(b), which reveals a strong contribution of very slow electrons as well. These observations, which we interpret as frustrated recombination in cluster nanoplasmas, may be of significant importance for the explanation of high charge states observed in FEL experiments [29,34,35].

In summary, use of the VMI technique in rare-gas cluster experiments under intense XUV irradiation provides novel information about the electron emission process. Angularresolved spectra show that the emission changes from a strongly anisotropic distribution for atoms to an almost isotropic distribution for large clusters, thereby underlining the increasing importance of collision processes. In addition, we have shown the first experimental evidence for the formation of very low kinetic energy electrons in the meV range that in accordance with previous theoretical work [23] are attributed to ionization from high-lying Rydberg 
states by the detector electric field. This suggests that the Rydberg states are occupied during the nanoplasma dissociation by three-body recombination processes. We expect the current findings to be important in a large range of excitation conditions and for finite systems in general. While our results are compatible with numerical calculations and FEL results, they do not agree with a previous experiment using a HHG source where $\mathrm{Xe}^{8+}$ was observed [24]. Indeed, we argue that with the reported intensity of $5 \times 10^{10} \mathrm{~W} / \mathrm{cm}^{2}$, it is very unlikely to obtain such high charge states.

*schuette@mbi-berlin.de

[1] K. Tiedtke et al., New J. Phys. 11, 023029 (2009).

[2] P. Emma et al., Nat. Photonics 4, 641 (2010).

[3] A. A. Sorokin, S. V. Bobashev, T. Feigl, K. Tiedtke, H. Wabnitz, and M. Richter, Phys. Rev. Lett. 99, 213002 (2007).

[4] B. Rudek et al., Nat. Photonics 6, 858 (2012).

[5] F. Krausz and M. Ivanov, Rev. Mod. Phys. 81, 163 (2009).

[6] T. Popmintchev et al., Science 336, 1287 (2012).

[7] W. Ackermann et al., Nat. Photonics 1, 336 (2007).

[8] J.-F. Hergott, M. Kovacev, H. Merdji, C. Hubert, Y. Mairesse, E. Jean, P. Breger, P. Agostini, B. Carré, and P. Salières, Phys. Rev. A 66, 021801 (2002).

[9] Y. Nabekawa, H. Hasegawa, E. J. Takahashi, and K. Midorikawa, Phys. Rev. Lett. 94, 043001 (2005).

[10] H. Mashiko, A. Suda, and K. Midorikawa, Opt. Lett. 29, 1927 (2004).

[11] E. J. Takahashi, P. Lan, O. D. Mücke, Y. Nabekawa, and K. Midorikawa, Nat. Commun. 4, 2691 (2013).

[12] H. Iwayama et al., J. Phys. B 43, 161001 (2010).

[13] U. Saalmann, C. Siedschlag, and J. M. Rost, J. Phys. B 39, R39 (2006).
[14] T. Fennel, K.-H. Meiwes-Broer, J. Tiggesbäumker, P.-G. Reinhard, P. M. Dinh, and E. Suraud, Rev. Mod. Phys. 82, 1793 (2010).

[15] M. Arbeiter and T. Fennel, Phys. Rev. A 82, 013201 (2010).

[16] C. Bostedt et al., Phys. Rev. Lett. 100, 133401 (2008).

[17] C. Bostedt, H. Thomas, M. Hoener, T. Möller, U. Saalmann, I. Georgescu, C. Gnodtke, and J. M. Rost, New J. Phys. 12, 083004 (2010).

[18] E. Ackad, N. Bigaouette, S. Mack, K. Popov, and L. Ramunno, New J. Phys. 15, 053047 (2013).

[19] B. Ziaja, T. Laarmann, H. Wabnitz, F. Wang, E. Weckert, C. Bostedt, and T. Möller, New J. Phys. 11, 103012 (2009).

[20] M. Arbeiter and T. Fennel, New J. Phys. 13, 053022 (2011).

[21] C. Gnodtke, U. Saalmann, and J.-M. Rost, Chem. Phys. 414, 65 (2013).

[22] A. Eppink and D. Parker, Rev. Sci. Instrum. 68, 3477 (1997).

[23] T. Fennel, L. Ramunno, and T. Brabec, Phys. Rev. Lett. 99, 233401 (2007).

[24] B. F. Murphy, K. Hoffmann, A. Belolipetski, J. Keto, and T. Ditmire, Phys. Rev. Lett. 101, 203401 (2008).

[25] K. Hoffmann, B. Murphy, N. Kandadai, B. Erk, A. Helal, J. Keto, and T. Ditmire, Phys. Rev. A 83, 059903(E) (2011).

[26] G. Gademann, F. Ple, P.-M. Paul, and M. J. J. Vrakking, Opt. Express 19, 24922 (2011).

[27] O. F. Hagena, Surf. Sci. 106, 101 (1981).

[28] M. Vrakking, Rev. Sci. Instrum. 72, 4084 (2001).

[29] H. Wabnitz et al., Nature (London) 420, 482 (2002).

[30] H. Iwayama et al., J. Phys. B 42, 134019 (2009).

[31] H. Thomas, C. Bostedt, M. Hoener, E. Eremina, H. Wabnitz, T. Laarmann, E. Plönjes, R. Treusch, A. R. B. de Castro, and T. Möller, J. Phys. B 42, 134018 (2009).

[32] D. Rolles, H. Zhang, Z. Pesić, J. Bozek, and N. Berrah, Chem. Phys. Lett. 468, 148 (2009).

[33] E. Ackad, N. Bigaouette, K. Briggs, and L. Ramunno, Phys. Rev. A 83, 063201 (2011).

[34] H. Thomas et al., Phys. Rev. Lett. 108, 133401 (2012).

[35] T. Gorkhover et al., Phys. Rev. Lett. 108, 245005 (2012). 